\title{
Tidal modulation of two-layer hydraulic exchange flows
}

\author{
L. M. Frankcombe ${ }^{1, *}$ and A. McC. Hogg ${ }^{1}$ \\ ${ }^{1}$ Research School of Earth Sciences, The Australian National University, Canberra, Australia \\ *now at: Institute for Marine and Atmosphere Research Utrecht, Department of Physics and Astronomy, Utrecht University, \\ The Netherlands
}

Received: 1 November 2006 - Published in Ocean Sci. Discuss.: 27 November 2006

Revised: 5 April 2007 - Accepted: 13 April 2007 - Published: 26 April 2007

\begin{abstract}
Time-dependent, two layer hydraulic exchange flow is studied using an idealised shallow water model. It is found that barotropic time-dependent perturbations, representing tidal forcing, increase the baroclinic exchange flux above the steady hydraulic limit, with flux increasing monotonically with tidal amplitude (measured either by height or flux amplitude over a tidal period). Exchange flux also depends on the non-dimensional tidal period, $\gamma$, which was introduced by Helfrich (1995). When tidal amplitude is characterised by the barotropic flux amplitude, exchange flux is a monotonic function of $\gamma$ as predicted by Helfrich (1995). However, the relationship between the (imposed) free surface amplitude and flux amplitude is complicated by reflections within the channel and by the baroclinic response of the two layer system, leading to a non-monotonic relationship between the height amplitude and $\gamma$.
\end{abstract}

\section{Introduction}

Flow of stratified water through ocean straits makes an important contribution to the evolution of ocean stratification, affecting global circulation and the local dynamics of estuaries and semi-enclosed basins. For example, exchange flow through the Strait of Gibraltar at the mouth of the Mediterranean Sea controls the salinity budget of the evaporative Mediterranean basin (Bray et al., 1995). Furthermore, the dense, saline outflow of water from Gibraltar can be detected as a distinct water mass across the North Atlantic (Sy, 1988; Harvey and Arhan, 1988). It follows that characterisation of flow through straits is an important problem, especially considering the difficulty most ocean and climate models face in resolving strait dynamics.

Correspondence to: L. M. Frankcombe

(1.frankcombe@phys.uu.nl)
Internal hydraulic theory can give a useful estimate of density-driven flow through straits in particular cases (Wood, 1970; Armi, 1986). This theory can be used to predict an upper bound for exchange flow through a strait (Armi and Farmer, 1987). However, this upper bound can be exceeded in cases where a time-dependent forcing, such as tidal flow, exists (Armi and Farmer, 1986).

Stigebrandt (1977) proposed a time-dependent solution for flow through straits which showed reasonable agreement with laboratory experiments. This theory was superseded by Armi and Farmer (1986), who formulated a quasi-steady solution based on hydraulically controlled solutions with a barotropic throughflow. The quasi-steady solution assumed that tidal variations were sufficiently slow so that hydraulic control was continually established; however, hydraulic control itself is not well defined in a time-dependent flow. Furthermore, Helfrich (1995) showed that tidal variations may exceed the frequency over which the quasi-steady solution is valid and introduced a nondimensional parameter, $\gamma$, the ratio of tidal period to the time taken for a baroclinic wave to traverse the strait. It is defined as

$\gamma \equiv \frac{T \sqrt{g^{\prime} H}}{l}$

where $T$ is the period of the wave, $g^{\prime}=g \Delta \rho / \rho$ is reduced gravity, $H$ is the total fluid height and $l$ is the length scale of the channel, i.e. the distance between the narrowest part of the channel (where channel width is $b_{0}$ ) and the point where channel width is $2 b_{0}$. Helfrich (1995) predicted that flux would depend upon both the dynamic strait length $\gamma$, and the amplitude of the tide, because higher frequency waves vary faster than the baroclinic flow can adjust. This prediction was consistent with simulations from a simple numerical model with a rigid lid. Helfrich also conducted laboratory experiments which showed that flux depends on $\gamma$, but that mixing and other effects act to reduce the flux. 


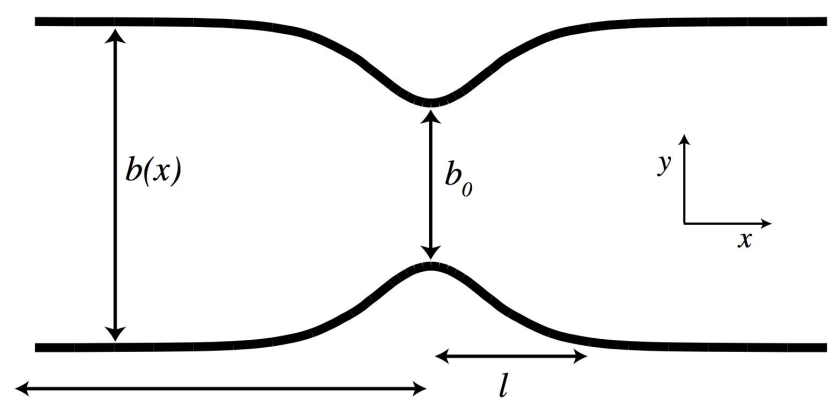

$L$

(a)

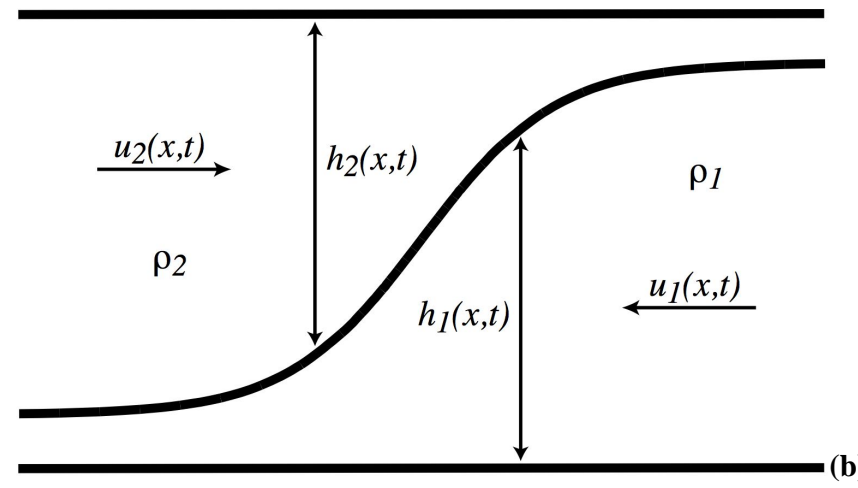

Fig. 1. Schematic showing (a) plan and (b) elevation view of the flow. Velocity in the lower layer $\left(u_{1}\right)$ is negative.

Additional experiments were conducted by Phu (2001) (reported by Ivey, 2004), in which both tidal amplitude and frequency were varied. It was found that exchange flux was strongly dependent on tidal amplitude, but that there was no systematic dependence upon tidal period, over a wide range of $2<\gamma<70$ (Ivey, 2004). This finding is inconsistent with the analytical predictions, numerical simulations and experiments of Helfrich (1995).

In this paper we take a different, numerical, approach with the goal of establishing the role of tidal period in exchange flows. We use a simple two-layer model of the shallow water equations to calculate the response of hydraulic exchange flows to time-dependent forcing. Barotropic forcing is induced at the boundaries and propagates into the domain as a free surface wave (unlike Helfrich's baroclinic numerical model which used a rigid lid). The waves modify the flux, which can be accurately measured, and the results are compared to the quasi-steady solution of Armi and Farmer (1986), the predictions of Helfrich (1995) and the experimental results of Phu (2001).

We begin the paper by outlining the model and boundary conditions in Sect. 2. Section 3 shows the results of the model, which are developed to span a wide parameter space in both the frequency and amplitude of time-dependent forcing. These results are discussed in Sect. 4, and application to geophysical flows is considered.

\section{The model}

\subsection{Shallow water equations}

The model used here is formulated to include the physics of time-dependent exchange flows with the minimum possible alterations to the steady hydraulic equations. We therefore solve the one-dimensional, hydrostatic, nonlinear shallow water equations for flow along a rectangular channel. The channel has length $2 L$ and variable width $b(x)$. We assume that flow occurs within two distinct immiscible fluid layers. The thickness of each layer is $h_{i}(x, t)-$ as shown in Fig. 1. The layers are assumed to have constant density $\rho_{i}$, and velocity $u_{i}(x, t)$ which depends upon horizontal, but not vertical, position.

The conservation of mass (or continuity) equation for each layer is given by

$\frac{\partial h_{i}}{\partial t}=-\frac{1}{b} \frac{\partial}{\partial x}\left(b u_{i} h_{i}\right)$.

The conservation of momentum equations are

$\frac{\partial u_{1}}{\partial t}+u_{1} \frac{\partial u_{1}}{\partial x}=-\frac{g}{g^{\prime}} \frac{\partial}{\partial x}\left(h_{2}+h_{1}\right)+\frac{\partial h_{2}}{\partial x}+\frac{1}{R e} \frac{\partial^{2} u_{1}}{\partial x^{2}}$

and

$\frac{\partial u_{2}}{\partial t}+u_{2} \frac{\partial u_{2}}{\partial x}=-\frac{g}{g^{\prime}} \frac{\partial}{\partial x}\left(h_{2}+h_{1}\right)+\frac{1}{R e} \frac{\partial^{2} u_{2}}{\partial x^{2}}$,

where the reduced gravity, $g^{\prime}$, is defined as follows:

$g^{\prime} \equiv \frac{g\left(\rho_{1}-\rho_{2}\right)}{\rho_{1}}$.

These equations have been written in non-dimensional form, where $h_{i}$ has been scaled by total fluid height $H, x$ by $\ell$ and $u_{i}$ by the baroclinic long wave speed $\sqrt{g^{\prime} H}$ (following Helfrich, 1995). Note that we have included a horizontal viscosity with a constant coefficient $A_{h}$, leading to a Reynolds number defined by $R e=\ell \sqrt{g^{\prime} H} / A_{h}$. Viscosity is required for numerical stability but is minimised in the simulations.

The solution of (2)-(4) under the assumption of a steady flow and small aspect ratio (i.e. $H \ll \ell$ ) may yield hydraulically controlled flow solutions (depending upon the boundary conditions at either end of the channel). The full timedependent equations may be solved numerically in a straightforward manner; but results are again dependent upon the correct boundary conditions.

\subsection{Boundary conditions}

The boundary conditions used for this model are the characteristic open boundary conditions based on the timeintegrating conditions proposed by Nycander and Döös (2003) and further developed for inertial flows by Nycander 
et al. (2006) ${ }^{1}$. These conditions require us to specify characteristic variables $a_{E}^{ \pm}$and $a_{I}^{ \pm}$at each of the open boundaries. The variables are defined as

$a_{E}^{ \pm}=\frac{1}{2}\left[h_{1}+h_{2} \pm\left(H_{1} u_{1}+H_{2} u_{2}\right) \sqrt{g^{\prime} / g}\right]$,

$a_{I}^{ \pm}=\frac{1}{2}\left[\left(H_{1} h_{2}-H_{2} h_{1}\right) \pm \sqrt{\frac{H_{1} H_{2}}{1-\Delta U^{2}}}\left(u_{2}-u_{1}\right)\right]$.

These equations are formulated by linearising the equations about a state with layer heights $H_{1}$ and $H_{2}$ (where $H=H_{1}+H_{2}$ ), and velocity difference $\Delta U$ between the layers.

The conditions are implemented as follows. Assume, for example, that the boundary is an $h$-point of a staggered grid. Then we must specify the inward travelling characteristic variables $a_{E}^{ \pm}$and $a_{I}^{ \pm}$. Having done that, the values of $h_{1}$ and $h_{2}$ at the boundary can be expressed in terms of $a_{E}^{ \pm}, a_{I}^{ \pm}$ and the values of $u_{1}$ and $u_{2}$ at the point just inside:

$$
\begin{array}{r}
h_{1}(\mp L)=2 H_{1} a_{E}^{ \pm}-2 a_{I}^{ \pm} \mp H_{1}\left(H_{1} u_{1}+H_{2} u_{2}\right) \sqrt{g^{\prime} / g} \\
\pm \sqrt{\frac{H_{1} H_{2}}{1-\Delta U^{2}}}\left(u_{1}-u_{2}\right),
\end{array}
$$

$$
\begin{array}{r}
h_{2}(\mp L)=2 H_{2} a_{E}^{ \pm}+2 a_{I}^{ \pm} \mp H_{2}\left(H_{1} u_{1}+H_{2} u_{2}\right) \sqrt{g^{\prime} / g} \\
\mp \sqrt{\frac{H_{1} H_{2}}{1-\Delta U^{2}}}\left(u_{1}-u_{2}\right) .
\end{array}
$$

The above conditions are suitable for sub-critical flow, but this model also needs to be able to simulate supercritical twolayer flow. The model is designed to switch to supercritical flow boundary conditions subject to a test on the criticality of the flow. There are two modes of supercritical flow (i.e. supercritical with respect to barotropic and baroclinic modes) and therefore two tests. The test for supercritical barotropic flow is based on the linear phase speed of a barotropic wave. When

$\frac{h_{1} u_{1}+h_{2} u_{2}}{h_{1}+h_{2}}>\sqrt{\left(h_{1}+h_{2}\right) g / g^{\prime}}$,

at the right hand end of the channel, flow is adjudged supercritical. There is an analogous condition at the left hand end of the channel. When this test is satisfied, the supercritical open boundary conditions are simply

$\frac{\partial h_{1}}{\partial x}=\frac{\partial h_{2}}{\partial x}=0$,

which replaces (7)-(8).

\footnotetext{
${ }^{1}$ Nycander, J., Hogg, A. M., and Frankcombe, L. M.: Open boundary conditions for nonlinear shallow water models, Ocean Modell., under review, 2006.
}

Internally supercritical flow is more complicated. Firstly, using linear internal wavespeeds to test for internal criticality, we obtain

$\frac{h_{2} u_{1}+h_{1} u_{2}}{h_{1}+h_{2}}>\sqrt{\frac{h_{1} h_{2}}{\left(h_{1}+h_{2}\right)^{2}}\left(\left(h_{1}+h_{2}\right)-\left(u_{1}-u_{2}\right)^{2}\right)}$,

at the right hand end of the channel (and an analogous condition for the left hand end). It should be noted that the RHS of this condition becomes imaginary when shear is strong, in which case the wavespeeds coalesce and waves are unstable. Therefore, we propose that a suitable test for criticality is simply

$\frac{h_{2} u_{1}+h_{1} u_{2}}{h_{1}+h_{2}}>\sqrt{\frac{h_{1} h_{2}}{\left(h_{1}+h_{2}\right)^{2}} \Theta}$,

where

$\Theta \equiv \max \left(0,\left(\left(h_{1}+h_{2}\right)-\left(u_{1}-u_{2}\right)^{2}\right)\right)$.

The boundary conditions for internally supercritical flow are found by assuming that the internal mode is captured primarily by interfacial height, and so we set

$\frac{\partial h_{1}}{\partial x}=0$,

with $h_{2}$ calculated using the addition of (7) and (8).

\subsection{Numerical implementation}

The domain is spatially discretised on a staggered grid. Velocity is calculated at the faces of the cells, while layer height is calculated at the centre of the cell. The equations may then be integrated in time using centred differences. The staggered grid is defined so that the near-boundary values of velocity can be explicitly calculated from $(3,4)$.

The temporal discretisation employs a leapfrog timestep scheme. Leapfrog timestepping routines can produce two diverging solutions. To eliminate this potential problem, data from different time levels are mixed every 1000 timesteps. The standard parameter set for the simulations is shown in Table 1.

\section{Results}

\subsection{Exchange flows}

The model is initialised with two constant depth, zero velocity layers. Layer depth variations, and hence velocity, are induced by specifying the characteristic boundary conditions, as seen in the series of snapshots in Fig. 2, which shows the development of the exchange flow. Internal waves propagate from either side of the domain, reaching the centre of the constriction after a nondimensional time of about 0.4 . By time 0.8 , hydraulic control is established at the centre 

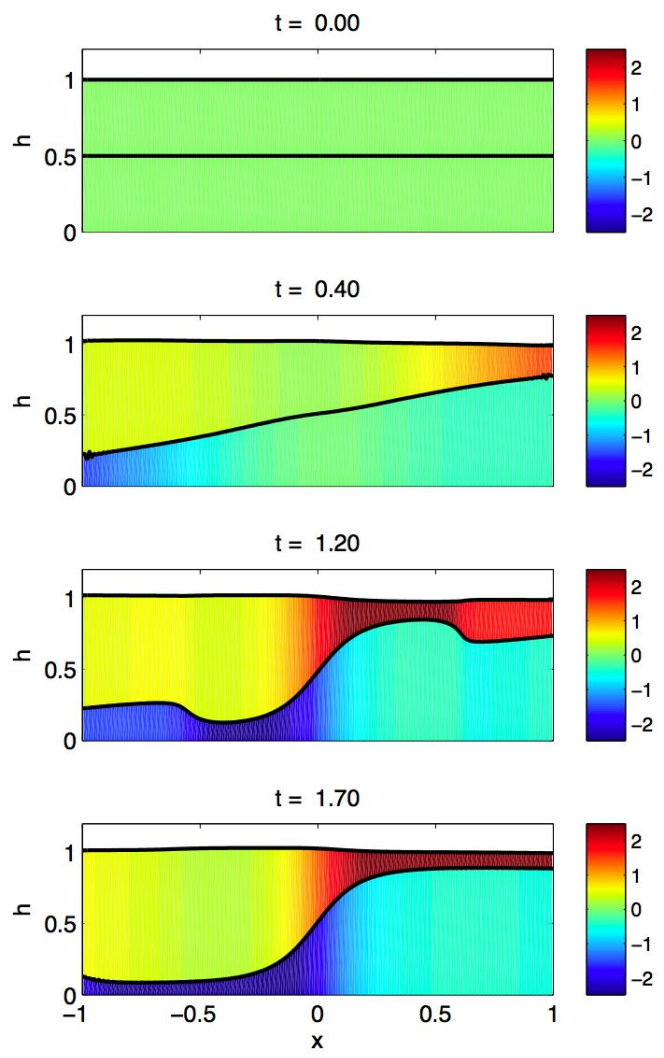
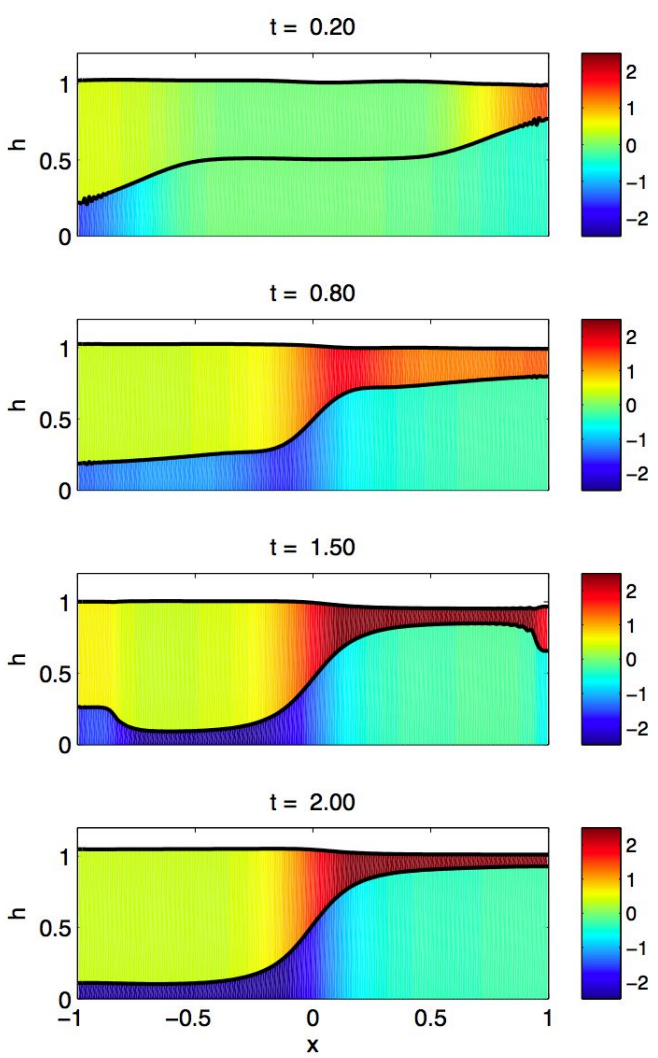

Fig. 2. Development of two layer flow (for a channel with $b_{\max }=3 b_{0}$ ). The colour scale shows dimensionless velocity.

Table 1. Standard physical and computational parameters for simulations. See Nycander et al. (2006) ${ }^{1}$ for an explanation for the values of the internal and external characteristics.

\begin{tabular}{|c|c|c|}
\hline Parameter & Value & Description \\
\hline $\operatorname{Re}$ & 13 & Reynolds number \\
\hline$b_{\max }$ & 2 & Maximum channel width (at $x= \pm L$ ) \\
\hline $\mathrm{g}^{\prime} / \mathrm{g}$ & 0.01 & Reduced gravity \\
\hline $\mathrm{L}$ & 1 & Channel length \\
\hline$\Delta \mathrm{t}$ & $1.2 \times 10^{-6} \mathrm{~s}$ & Timestep \\
\hline $\mathrm{n}$ & 201 & Number of gridpoints \\
\hline$\Delta \mathrm{x}$ & 0.01 & Gridlength \\
\hline$a_{e}^{+}$ & 0.017 & External BC coefficient at LHS \\
\hline$a_{e}^{-}$ & 0.0 & External BC coefficient at RHS \\
\hline$a_{i}^{+}$ & 0.07028 & Internal BC coefficient at LHS \\
\hline$a_{i}^{-}$ & -0.07028 & Internal BC coefficient at RHS \\
\hline
\end{tabular}

of the channel, and features resembling hydraulic jumps are formed. It should be noted that the model equations are not designed to simulate the nonhydrostatic or dispersive dynamics of these jumps, and we are therefore unable to accurately model flows in which shocks are present in the steady state. However, these features propagate out of the domain, leaving a final steady state matching the two-layer maximal hydraulic exchange flow solution, in which flow is internally supercritical at both ends of the channel. It is notable that there are no significant reflections from the characteristic open boundary conditions during this adjustment process - this issue is examined more closely by Nycander et al. (2006) ${ }^{1}$.

Time dependence is introduced to this flow by sinusoidally varying the left boundary condition coefficient, $a_{e}^{+}$, with a period of $\gamma=0.2$. This simulates a barotropic wave entering from the left, as seen in Fig. 3. The incoming wave travels towards the centre of the channel where it interacts with the contraction, causing reflections (both baroclinic and barotropic) to travel back to the left, while the original barotropic wave, which now includes a small baroclinic component, continues to the right.

Barotropic flux as a function of time at the left-hand boundary is shown in Fig. 4 (flux is not completely symmetric around zero because of a small barotropic contribution to the mean flow). The system takes several periods for the oscillations to become regular, as instantaneous flux is modulated by waves reflected off the contraction. For this reason, all results in the following sections were calculated after the initial adjustment had occurred. 

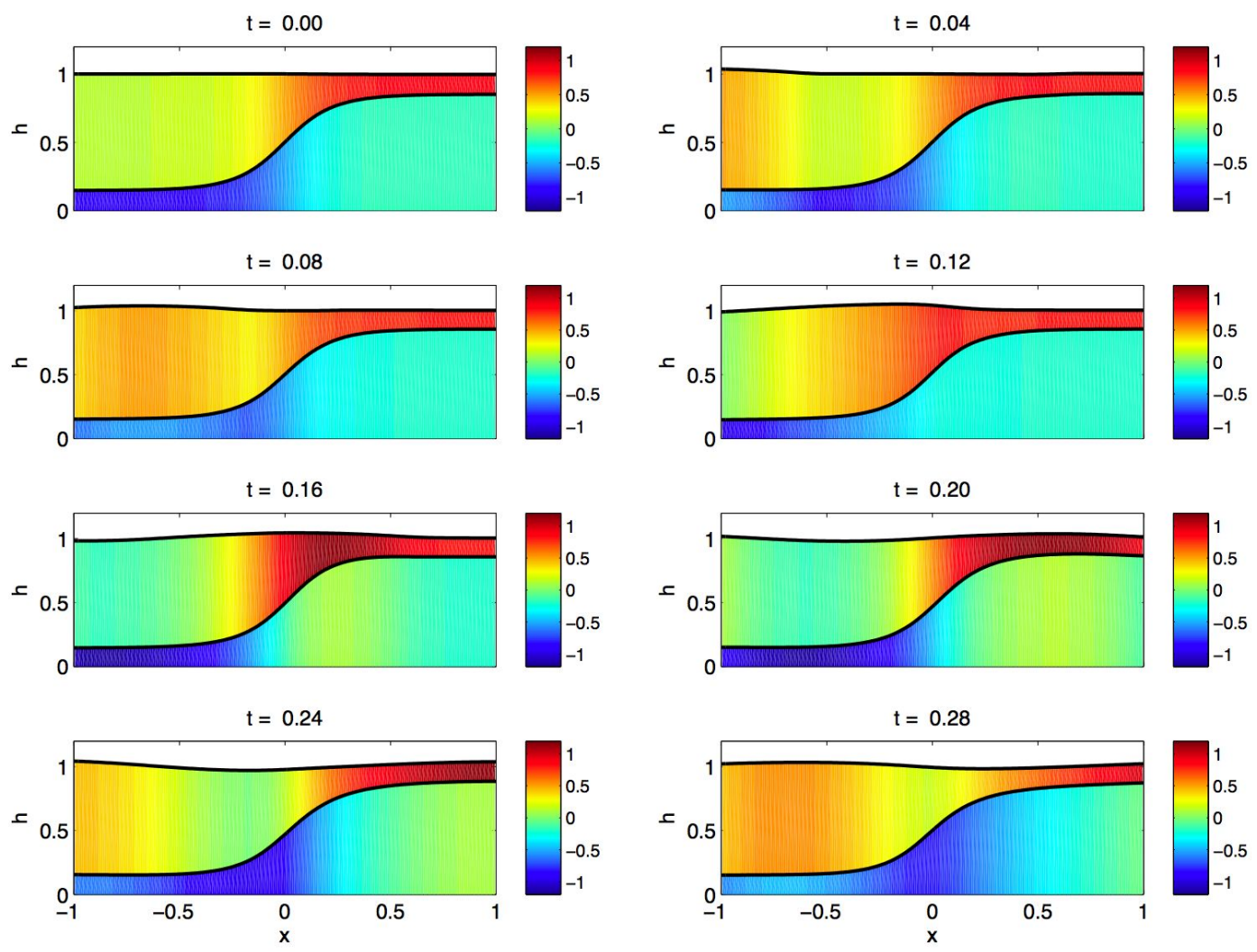

Fig. 3. Propagation of a wave through the channel. The colour scale shows dimensionless velocity.

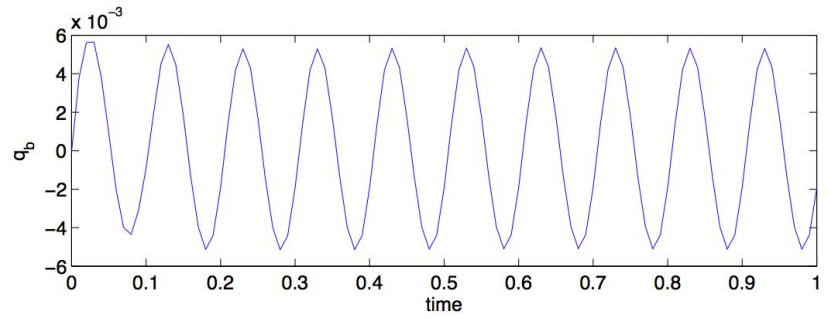

Fig. 4. Barotropic flux, $q_{b}$, measured at the left hand boundary.

\subsection{Exchange flux in time-dependent systems}

The quantity of primary interest in these simulations is the baroclinic flux of volume (or mass) exchanged, $q_{c}$. We quantify $q_{c}$ by calculating the total volume flux in each layer as a function of time, integrating over a tidal period and averaging the (absolute value of the) two layer fluxes. The baroclinic exchange flux is then proportional to the net exchange of mass (or passive tracer) through the channel. Figure 5 shows the exchange flux anomaly as a function of nondimensional period $\gamma$, for three cases with the same imposed amplitude but differing reduced gravity and channel length. The baroclinic flux anomaly, $q_{c}^{\prime}=q_{c}-\overline{q_{c}}$ (where $\overline{q_{c}}$ is the flux for the steady solution) has been scaled by the quasi-steady flux, cal-

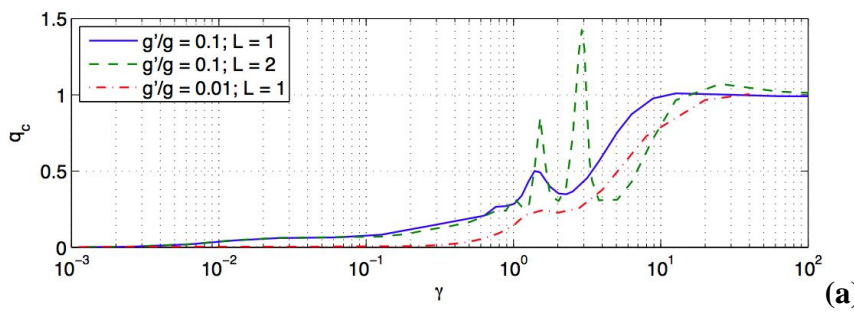

Fig. 5. Baroclinic exchange flux anomaly, $q_{c}^{\prime}$, for three simulations with different values of $g^{\prime}$ and $L$, scaled by the quasi-steady flux.

culated numerically from a series of steady simulations and sets a theoretical upper limit for the flux.

In the small period (small $\gamma$ ) limit the flux anomaly approaches zero (the steady hydraulic limit), and in the large $\gamma$ limit it approaches the quasi-steady solution in each of the three cases shown. These limits are consistent with the predictions of Helfrich (1995); however here the flux is not a monotonically increasing function of $\gamma$. Instead, there are additional local maxima (the largest being at $\gamma \approx 3$ ) which sometimes exceed the quasi-steady limit. These peaks are greatest when $g^{\prime}$ is large, and when the domain length $L$ is increased (dashed line in Fig. 5). 


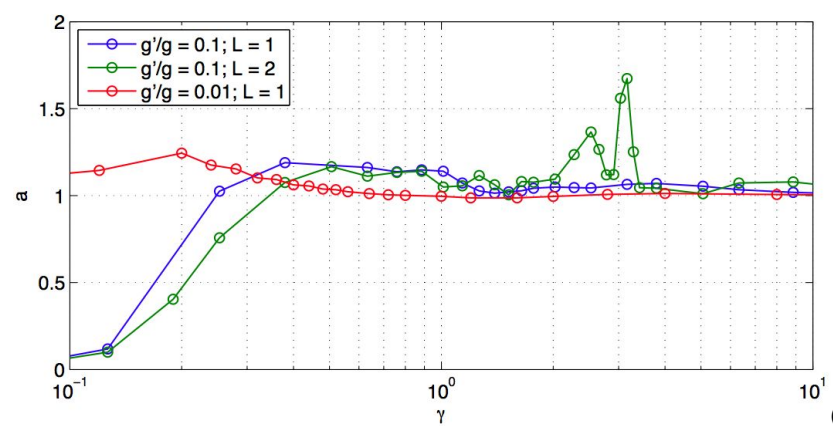

(a)

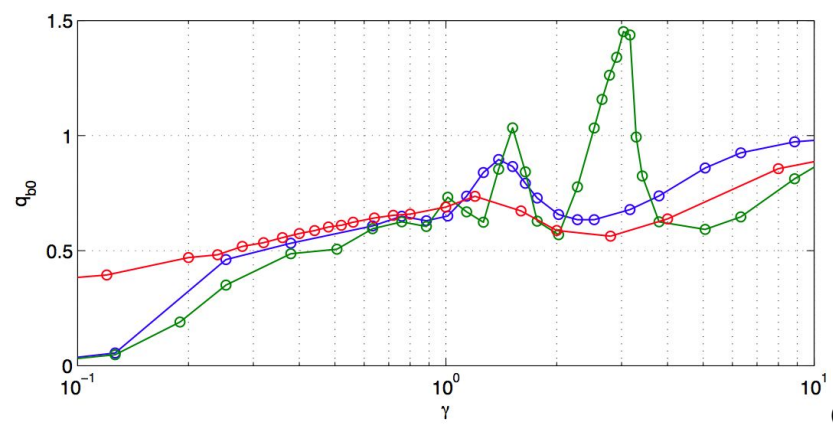

(b)

Fig. 6. (a) Height amplitude and (b) flux amplitude as a function of $\gamma$ for three different cases.

The peaks in baroclinic flux anomaly may be due to either resonance in the channel between the open boundary and the contraction, or to the internal dynamics of the two-layer flow. We test for both of these effects by calculating the amplitude of the barotropic response at the centre of the channel in two ways. Firstly, $a$ represents the peak-to-trough amplitude of fluid height over the tidal cycle (normalised by mean fluid height $H$ ). We refer to this as the height amplitude and it is analogous to the method used by Phu (2001) to characterise tidal amplitude. Variations in height amplitude with $\gamma$ (when imposed amplitude is constant) indicate that resonant reflections between the contraction and the open boundary may be acting to enhance the amplitude of waves.

The second measure of amplitude is the barotropic flux amplitude, similar to Helfrich's $q_{b 0}$ parameter, which we define as the peak-to-trough amplitude of the barotropic flux, $q_{b}$,

$q_{b} \equiv b\left(h_{1} u_{1}+h_{2} u_{2}\right)$

where flow variables have been non-dimensionalised as in Sect. 2. Variations in $q_{b 0}$ will also occur in response to resonant reflections. In addition we note the possibility that the internal dynamic response of the two-layer flow may alter this measure of amplitude.

The measurements of height and flux amplitude as a function of $\gamma$ (for constant imposed amplitude) are plotted in Fig. 6. These measurements help to clarify the source of peaks in the baroclinic flux curves. The peak in the green

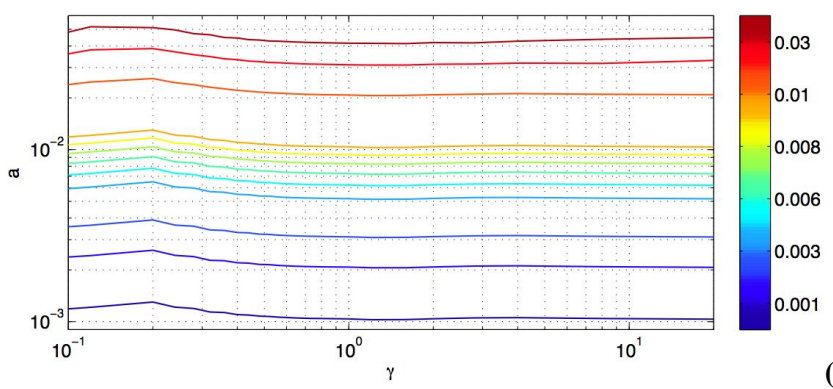

(a)

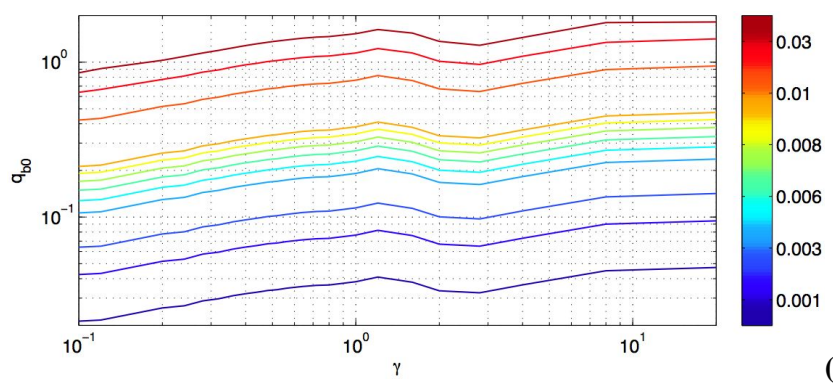

(b)

Fig. 7. Observed (a) height and (b) flux amplitudes are plotted against $\gamma$ for different imposed $a_{e}^{+}$amplitudes.

curve (long channel case) at $\gamma=3$ is seen in both height and flux amplitude, indicating that this is a resonant effect. This occurs in the long channel case because wave steepening leads to bores which enhance wave reflection off the open boundary. The resonant frequency, $\gamma=3$, is consistent with a baroclinic wave with half wavelength equal to the distance between the open boundary and the maximum rate of change of the channel width (at $x \approx-0.5$ ). This implies that resonance is due to the baroclinic wave reflected from the contraction, which steepens and interacts with the numerical condition at the open boundary. Similarly the second peak (at $\gamma \approx 1.5$ ) is consistent with a baroclinic wave with wavelength equal to the distance between the open boundary and the maximum rate of change of the channel width. Small deviations in the green curve in Fig. 6 at $\gamma \approx 1.5$ also indicate that resonance may be having an effect here. Note that exact prediction of the resonant period is complicated by the role of the mean flow in governing wavespeeds.

At higher frequencies $(\gamma<1)$, the height amplitude increases slightly in all three cases. This is due to nonlinear steepening of the high frequency waves, rather than reflections.

The response of the flux amplitude as a function of wave period shows a consistent peak at $\gamma \approx 1.5$ for all three cases shown here. This peak is not present in the height amplitude signal for the cases with $L=1$, indicating that although resonance may still be occuring in these cases, it may not be the main cause of the peaks. Thus, we infer that this peak describes a maximum in the barotropic forcing which is instead due to the internal dynamic response of the critical flow 

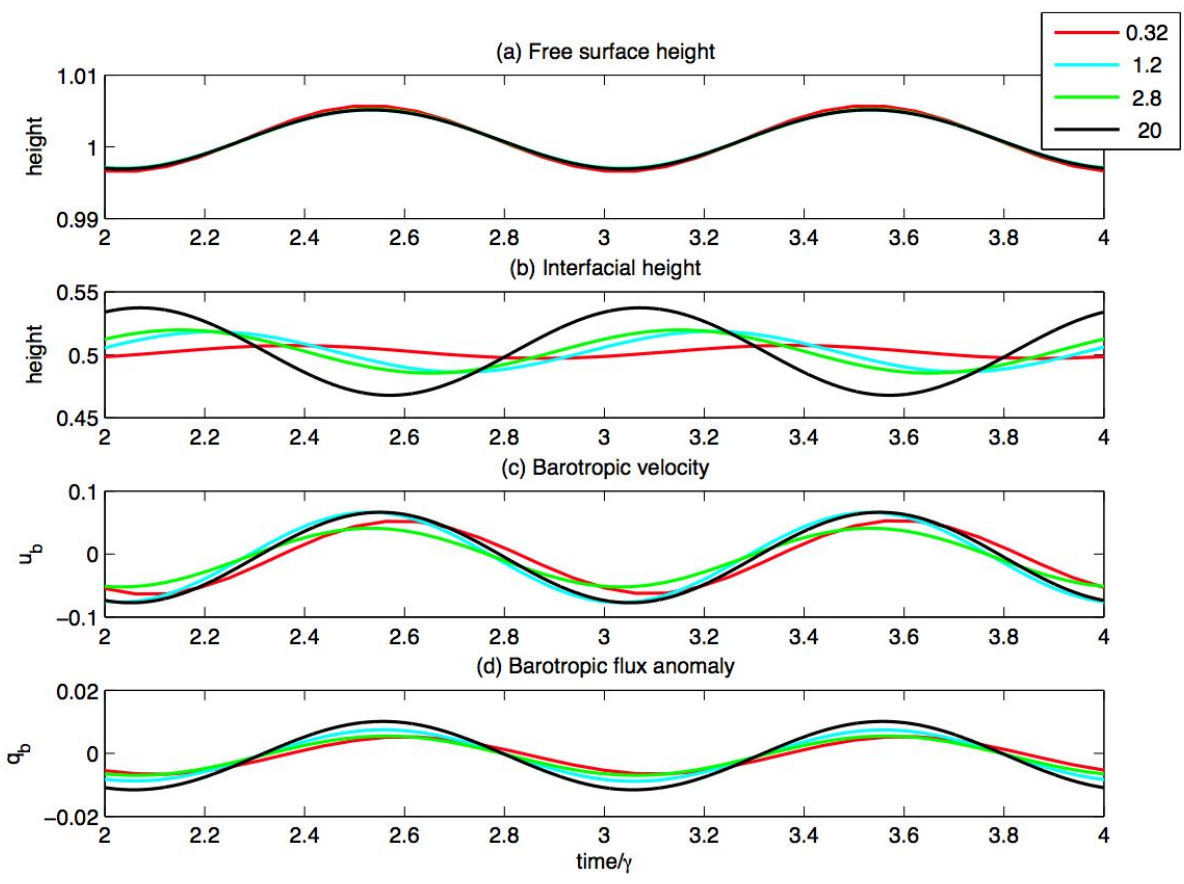

Fig. 8. (a) Free surface height; (b) Interfacial height and (c) barotropic velocity $u_{b}$ and (d) barotropic flux anomaly for four simulations with the same imposed amplitude but differing values of $\gamma$. Note that the time axis has been scaled by $\gamma$ and time-shifted so that free surface height perturbations are in phase.

in the contraction region, and investigate more closely using a particular case.

\subsection{Internal response of the flow}

We have conducted a large number of additional simulations in which the amplitude and period of imposed tidal forcing is varied using the parameters $g^{\prime} / g=0.01 ; L=1$. This case is chosen because resonant effects are weak, allowing us to focus on the internal dynamics of the flow. Figure 7 a shows the height amplitude measured at the centre of the channel for different values of amplitude imposed at the boundary. The response of height amplitude for a wide range of imposed amplitude is consistent with Fig. 6a. For $\gamma \geq 1$ the lines of constant imposed amplitude are flat, while there is a weak dependence of height amplitude on tidal period for $\gamma<1$, indicating the role of nonlinear wave steepening at these higher frequencies. However, resonance, which is observed clearly for the long channel case, is not obvious here.

Figure $7 \mathrm{~b}$ shows that the frequency dependence of the flux amplitude response occurs regardless of the value of imposed amplitude (note the logarithmic scale on the y-axis). This effect peaks at $\gamma \approx 1.5$, with an additional trend in flux amplitude with period. Thus, flux amplitude is not simply related to imposed amplitude or observed height amplitude. This result is particularly significant given that Helfrich's (1995) results are couched in terms of flux amplitude rather than the (more commonly measured) height amplitude.
The frequency dependence of the barotropic flux amplitude is analysed to greater depth using four particular examples in Fig. 8. Figure 8a shows the evolution of the free surface at the centre of the channel, where the time axis on this figure has been scaled by $\gamma$, and a time-shift applied so that each example is in phase. The free surface heights change very little for the different values of $\gamma$, consistent with Fig. 7a. However, the interfacial height evolution (Fig. 8b) does change with tidal period. The black line shows a large $\gamma$ case (close to the quasi-steady limit) for which interface height anomalies are $180^{\circ}$ out of phase from the the free surface height and nearly 10 times the magnitude. This indicates that the period is sufficiently long that the internal interface can respond to the free surface. For the shortest period case $(\gamma=0.32)$ the interfacial response is weak in magnitude and almost in phase with the surface forcing, indicating that the interface barely has time to respond to the tide. The two cases with intermediate values of $\gamma$ produce an interfacial response $90^{\circ}$ out of phase with surface height.

We show a measure of barotropic velocity, defined simply as

$u_{b}=\frac{1}{2}\left(u_{1}+u_{2}\right)$

in Fig. 8c. The velocity is generally in phase with surface height, however a slight phase lag and reduction in amplitude occurs at $\gamma=0.32$ and 2.8 (but not for the $\gamma=1.2$ case).

The barotopic flux anomaly (Fig. 8d) shows how surface height, interfacial response and barotropic velocity conspire 


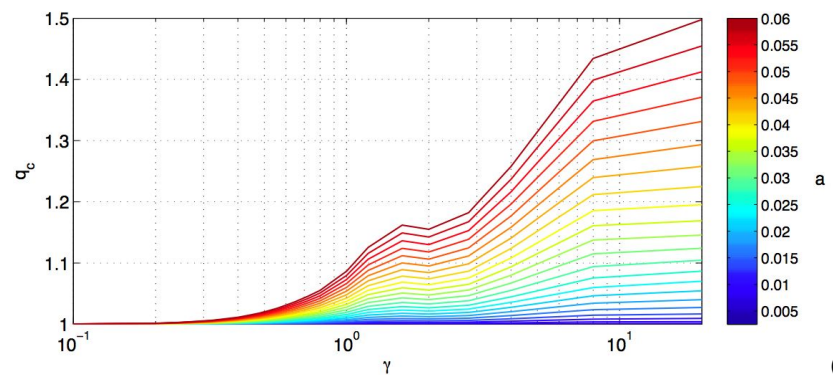

(a)

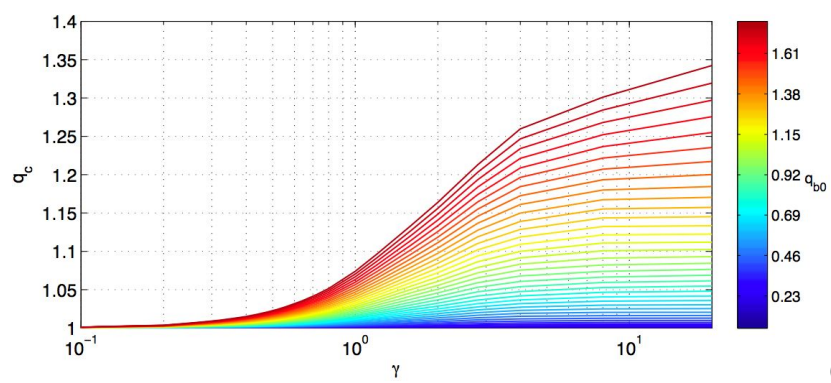

(b)

Fig. 9. Baroclinic flux, $q_{c}$ (normalised by the steady flux), plotted against $\gamma$ with (a) measured height amplitude and (b) measured flux amplitude in colour.

to produce larger flux amplitude at large $\gamma$, and the local maxima which occurs at $\gamma \approx 1.5$. We clarify the relative roles of these processes by writing layer velocity as

$u_{1} \approx-\bar{u}+u_{b}$,

$u_{2} \approx \bar{u}+u_{b}$,

where $\bar{u}$ is the (known) steady velocity. Also write layer depths as a mean perturbation

$h_{i}=\overline{h_{i}}+h_{i}^{\prime}$

allowing Eq. (15) to be written

$q_{b} \approx b\left(\bar{u}\left(\overline{h_{2}}-\overline{h_{1}}\right)+\bar{u}\left(h_{2}^{\prime}-h_{1}^{\prime}\right)+u_{b}\left(\overline{h_{2}}+\overline{h_{1}}\right)+u_{b}\left(h_{2}^{\prime}+h_{1}^{\prime}\right)\right)$.

We simplify this equation by assuming $\overline{h_{1}}=\overline{h_{2}}=0.5, b=0.5$ and $\bar{u}=0.5$ from the hydraulic solution, and that the last term, which is the product of two small quantities, can be ignored,

$q_{b} \approx 0.25\left(h_{2}^{\prime}-h_{1}^{\prime}\right)+0.5 u_{b}$.

The layer depth variations can be divided into surface, $h_{t}^{\prime}=h_{1}^{\prime}+h_{2}^{\prime}$, and interfacial height variations, $h_{i}^{\prime}=h_{1}^{\prime}$, and the barotropic velocity modelled as being a direct response to surface height perturbations, i.e.

$\frac{\partial u_{b}}{\partial t}=-\frac{g}{g^{\prime}} \frac{\partial h_{t}^{\prime}}{\partial x}$.

Assuming sinusoidal waves we find

$u_{b} \approx 10 h_{t}^{\prime}$.

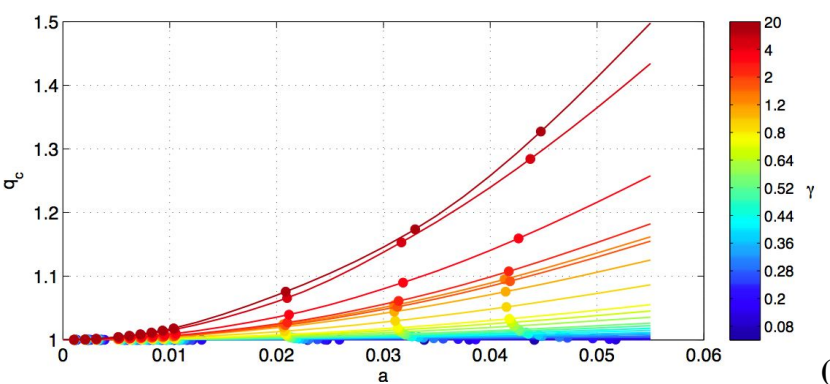

(a)

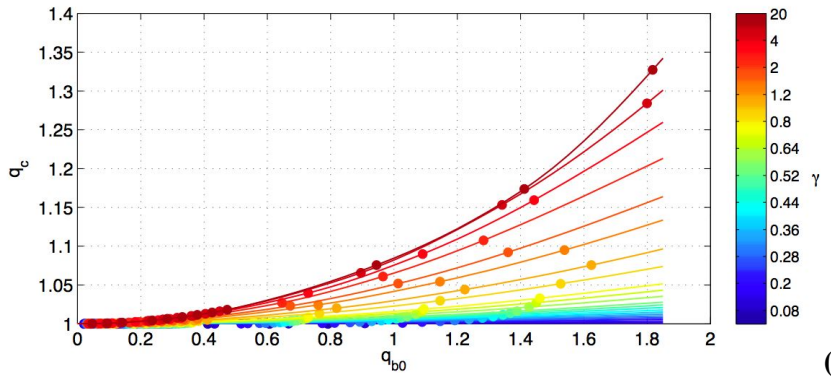

(b)

Fig. 10. Baroclinic flux, $q_{c}$ (normalised by the steady flux), plotted against (a) measured height amplitude and (b) measured flux amplitude, with $\gamma$ in colour. Solid circles show the original points from which the other points have been interpolated.

This we write

$q_{b} \approx-0.5 h_{i}^{\prime}+5.25 h_{t}^{\prime}$.

Equation (19) explains the major features observed in Fig. 8. At large $\gamma$, interfacial height is out of phase with surface height, and larger in magnitude, producing enhanced barotropic flux. At $\gamma=2.8$, barotropic velocity is smaller than predicted by Eq. (18) causing a local minima in flux amplitude. These two different processes combine to produce the the observed flux amplitude variation.

\subsection{Flux as a function of observed amplitudes}

For each of the simulations across a large range in tidal period and amplitude, measurements of baroclinic flux have been interpolated onto lines of (a) constant height amplitude, and (b) constant flux amplitude. These results are plotted as a function of $\gamma$ in Fig. 9 (normalised by the steady hydraulic flux). Figure 9a shows a peak at $\gamma=1.5$, indicating that height amplitude is not a good predictor of the tidal effect on exchange flows in this model, since it does not take the effect of resonance or the internal dynamics into account. For cases with greater amplitude and stronger stratification, such as the dashed lines in Fig. 5, the height amplitude is a very poor descriptor of the tidal effects. Figure $9 b$, on the other hand, clearly shows a smooth monotonic transition of flux with $\gamma$ when flux amplitude is used as the metric. These results are consistent with predictions of Helfrich (1995), and 
demonstrate that the internal response of the flow to free surface variations described in the previous section is additional to the phenomena documented by Helfrich. Thus, flux amplitude is a more useful predictor of the baroclinic response to tidal forcing than height amplitude in this model, because the resonance and internal dynamics are included.

The results are re-plotted in Fig. 10, showing amplitude vs flux for each of the above cases. The panels, showing baroclinic flux as a function of height and flux amplitude respectively, both show a tendency for flux to increase with amplitude.

\section{Discussion and conclusions}

The results of these simulations confirm that time-dependent forcing leads to an increase in exchange flux compared with the steady state case. Tidal amplitude, as measured by either height or flux amplitude, has a strong effect on flux, with flux increasing monotonically with amplitude for a fixed value of $\gamma$.

Flux also depends on the nondimensional tidal period $\gamma$. When $\gamma$ is very small, the system doesn't have time to respond to changes in forcing and the interfacial height variations is in phase with the variations of the surface height. The baroclinic flux is then equal to the hydrostatic limit. When $\gamma$ is large the system approaches the quasi-steady limit of Armi and Farmer (1986), where the flow is always controlled but the quadratic correlation between layer depth and velocity, coupled with the control condition, result in increased flux. In this case the interfacial height variations are $180^{\circ}$ out of phase with the variations in surface height. These limits are entirely consistent with those of Helfrich (1995).

Helfrich also predicts a smooth transition between these two limits when the barotropic flux amplitude, $q_{b 0}$ is held constant. Our results confirm this prediction, however we find that there is not a simple relationship between the barotropic flux amplitude and a tidal amplitude based on free surface height variations. Instead, the resonance and internal dynamics of the interfacial response and barotropic velocity modulate the flux. The result is that, for a given imposed amplitude, the barotropic (and hence baroclinic) flux depends strongly upon tidal period, upon the density difference between the two layers, and upon channel geometry.

For some parameter regimes we observed strong resonance caused by reflection of baroclinic bores at the open boundary. The resonant problem is a numerical artefact, but does indicate the difficulty involved in constructing numerical models of hydraulic flows.

These observations may explain some outstanding discrepancies between previous studies on this topic. Phu (2001) (reported by Ivey, 2004) used the height amplitude to characterise tidal strength and found no systematic dependence upon $\gamma$ over a wide range in parameter space, while Helfrich (1995) found a strong dependence of flux amplitude on $\gamma$. The height amplitude formalism used by Phu (2001) to characterise tidal strength may have been distorted by the baroclinic response, particularly for large amplitude waves. In addition, the solid wall at the end of Phu's experimental tank is likely to have amplified reflections within the reservoirs, further complicating measurements.

These results are likely to be relevant to the effect of tides in straits which border marginal seas. Such straits are listed by Helfrich (1995), and include the Bosphorus $\left(g^{\prime} / g=0.012, \gamma=3.1, q_{b 0}=0.25\right)$, Gibraltar $\left(g^{\prime} / g=0.002\right.$, $\left.\gamma=5.3, q_{b 0}=0.6\right)$ and Bab al Mandab $\left(g^{\prime} / g=0.0015, \gamma=0.5\right.$, $\left.q_{b 0}=0.3\right)$. Each of these straits is in a parameter regime in between the steady and quasi-steady limits, and close to the regime in which internal dynamics may modulate the flux.

It follows from this analysis that the best metric to describe geophysical time-dependent strait flows is the barotropic flux amplitude. However, measurements of tidal range are easiest to quantify in terms of height amplitude, rather than flux. Moreover, since we have shown that the internal response can modulate the barotropic flux, it would appear that an understanding of the time-dependent internal dynamics of the flow is required to exactly predict the time-dependent component of the flux. The need for such detailed knowledge of the flow eliminates the major advantages obtained by applying the hydraulically controlled solution to estimates of flux through a strait.

Acknowledgements. J. Nycander contributed significantly to this work, in assisting with the implementation of the boundary conditions and commenting on early drafts of this manuscript. The authors would like to thank two anonymous referees who contributed substantially to improving the first draft of this paper. LMF was funded by an A. L. Hales Scholarship, and AMH acknowledges the support of an Australian Research Council Postdoctoral Fellowship (DP0449851). This work was supported by the National Facility of the Australian Partnership for Advanced Computing.

Edited by: J. M. Huthnance

\section{References}

Armi, L.: The hydraulics of two flowing layers with different densities, J. Fluid Mech., 163, 27-58, 1986.

Armi, L. and Farmer, D. M.: Maximal two-layer exchange through a contraction with barotropic net flow, J. Fluid Mech., 164, 27$51,1986$.

Armi, L. and Farmer, D. M.: A Generalization of the concept of Maximal Exchange in a Strait, J. Geophys. Res., 92, 14 679$14680,1987$.

Bray, N., Ochoa, W. J., and Kinder, T.: The role of the interface in exchange through the Strait of Gibraltar, J. Geophys. Res., 100, 10755-10 776, 1995.

Harvey, J. and Arhan, M.: The Water Masses of the Central North Atlantic in 1983-84, J. Phys. Oceanogr., 18, 1855-1875, 1988.

Helfrich, K. L.: Time-dependent two-layer hydraulic exchange flow, J. Phys. Oceanogr., 25, 359-373, 1995. 
Ivey, G. N.: Stratification and mixing in sea straits, Deep-Sea Res. II, 51, 441-453, 2004.

Nycander, J. and Döös, K.: Open boundary conditions for barotropic waves, J. Geophys. Res., 108(C5), doi:10.1029/2002JC001 529, 2003.

Phu, A.: Tidally forced exchange flows, Honours thesis, University of Western Australia, 2001.
Stigebrandt, A.: On the effects of barotropic current fluctuations on the two-layer transport capacity of a constriction, J. Phys. Oceanogr., 7, 118-122, 1977.

Sy, A.: Investigation of large-scale circulation patterns in the Central North Atlantic: the North Atlantic Current, the Azores Current, and the Mediterranean Water plume in the area of the MidAtlantic Ridge, Deep Sea Res., 35, 383-413, 1988.

Wood, I. R.: A lock exchange flow, J. Fluid Mech., 42, 671-687, 1970. 